OPEN

SUBJECT AREAS:

EMBRYONIC STEM CELLS

T CELLS

Received

2 November 2014

Accepted

24 March 2015

Published

5 June 2015

Correspondence and requests for materials should be addressed to L.L. (laijun.lai@uconn. edu)

\section{Efficient in vitro generation of functional thymic epithelial progenitors from human embryonic stem cells}

Min Su ${ }^{1,2}$, Rong Hu' ${ }^{1}$ Jingiun Jin ${ }^{3}$, Yuan Yan' ${ }^{1}$ Yinhong Song ${ }^{1}$, Ryan Sullivan' \& Laijun Lai ${ }^{1,4}$

\begin{abstract}
${ }^{1}$ Department of Allied Health Sciences, University of Connecticut, Storrs, CT, ${ }^{2}$ Guiyang Medical College, Guizhou, China, ${ }^{3}$ Fujian Academy of Medical Sciences, China, ${ }^{4}$ University of Connecticut Stem Cell Institute, University of Connecticut, Storrs, CT.
\end{abstract}

Thymic epithelial cells (TECs) are the major components of the thymic microenvironment for $\mathrm{T}$ cell development. TECs are derived from thymic epithelial progenitors (TEPs). It has been reported that human ESCs (hESCs) can be directed to differentiate into TEPs in vitro. However, the efficiency for the differentiation is low. Furthermore, transplantation of hESC-TEPs in mice only resulted in a very low level of human T cell development from co-transplanted human hematopoietic precursors. We show here that we have developed a novel protocol to efficiently induce the differentiation of hESCs into TEPs in vitro. When transplanted into mice, hESC-TEPs develop into TECs and form a thymic architecture. Most importantly, the hESC-TECs support the long-term development of functional mouse T cells or a higher level of human $\mathrm{T}$ cell development from co-transplanted human hematopoietic precursors. The hESC-TEPs may provide a new approach to prevent or treat patients with $\mathrm{T}$ cell immunodeficiency.

cells play a critical role in the immune system, providing protection against infections and cancers. The thymus is the primary organ for $\mathrm{T}$ cell generation. $\mathrm{T}$ cell development in the thymus depends on the thymic microenvironment, of which TECs are the major component ${ }^{1-3}$. TECs can be divided into cortical TECs (cTECs) and medullary TECs (mTECs); the former mediate positive selection; and the latter are involved in the negative selection by which potentially autoreactive $\mathrm{T}$ cells are deleted. The relevance of TECs to $\mathrm{T}$ cell development is demonstrated by the fact that abnormal TEC development results in autoimmunity and immunodeficiency ${ }^{1-3}$. Furthermore, a decrease in TEC number results in a reduced number of T cells ${ }^{4}$.

Studies have shown that TECs undergo degeneration over time, which is believed to be one of the major factors responsible for age-associated thymic involution ${ }^{2,5-7}$. Moreover, TECs can be injured by radiation, chemotherapy, infection, and graft-versus-host disease following bone marrow (BM) transplantation ${ }^{8-10}$. Therefore, approaches to restore or regenerate TECs should also result in enhanced generation of $\mathrm{T}$ cells.

We have reported that mESCs can be selectively induced to differentiate into TEPs in vitro ${ }^{11,12}$. When transplanted into mice, the mESC-TEPs further develop into cTECs and mTECs, reconstitute normal thymic architecture, and promote $\mathrm{T}$ cell regeneration, leading to an increased number of functional $\mathrm{T}$ cells in peripheral lymphoid organs ${ }^{11,12}$.

Recently, it has been reported that hESCs can also be induced to differentiate into TEP-like cells in vitro ${ }^{13-15}$. However, generation of hESC-TEPs by these protocols lacks efficiency. Furthermore, although hESC-TEPs in nude mice further developed into TECs that supported mouse T cell development, a progressive decline in the number of $\mathrm{T}$ cells was observed, and $\mathrm{T}$ cell regeneration was not sustained over 22 weeks ${ }^{13}$. In addition, only one report showed that hESC-TECs could support human T cell development from co-transplanted human hematopoietic precursors, but the percentage of newly generated human $\mathrm{T}$ cells in the peripheral blood of the recipient mice was very low $^{14}$.

Here we report a new protocol for directing the differentiation of hESCs into TEPs in vitro. The hESC-TEPs, when transplanted into athymic nude mice, develop into both types of TECs that support a long-term generation of functional T cells. Most importantly, the hESC-TECs can also support a higher level of functional human $\mathrm{T}$ cell development from co-transplanted human hematopoietic precursors.

\section{Results}

Inducing the differentiation of hESCs into TEPs in vitro. Figure $1 \mathrm{~A}$ is a schematic of our differentiation protocol. Because the epithelial compartment of the thymus originates from the endoderm ${ }^{1,2,16}$, we first 

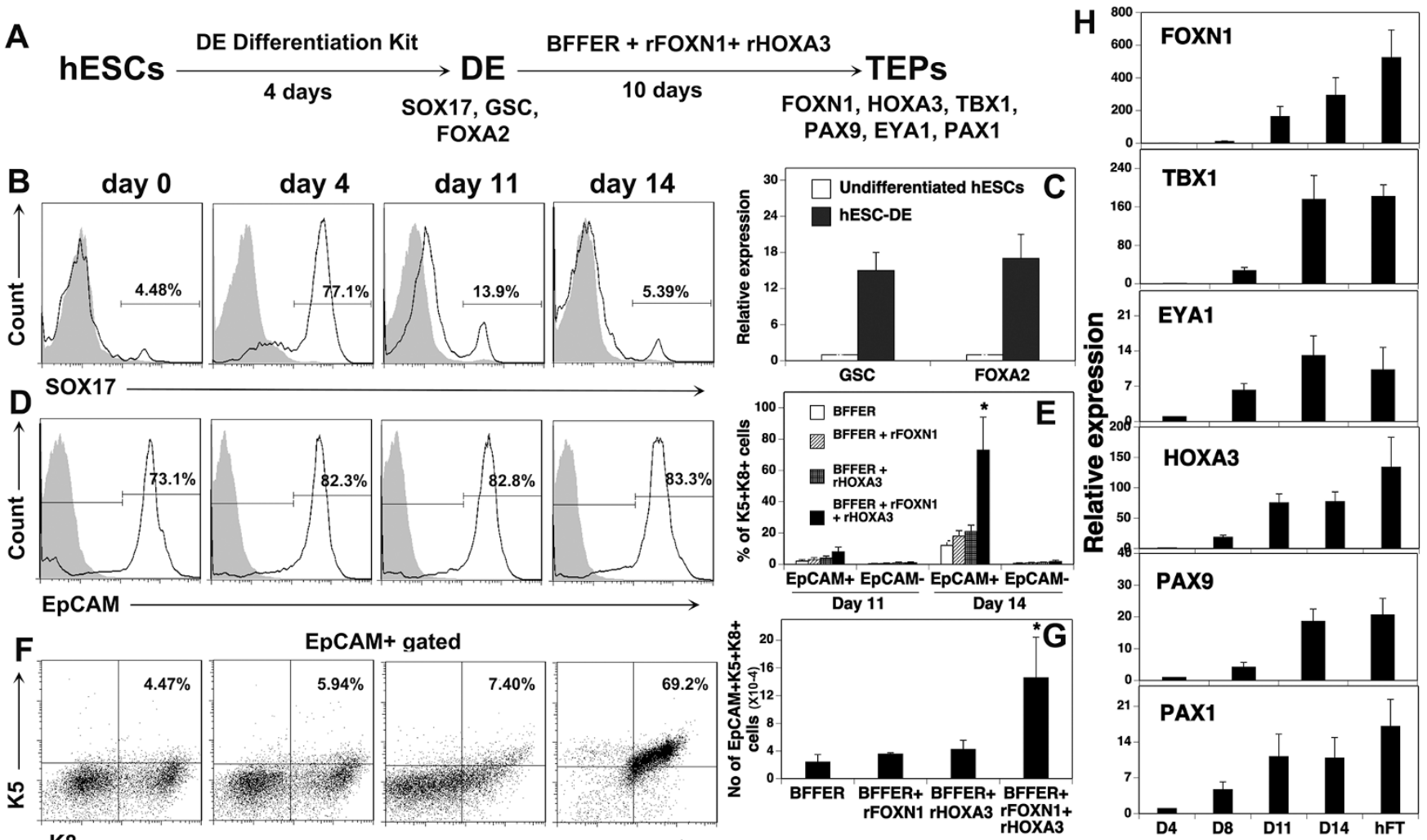
SOX17

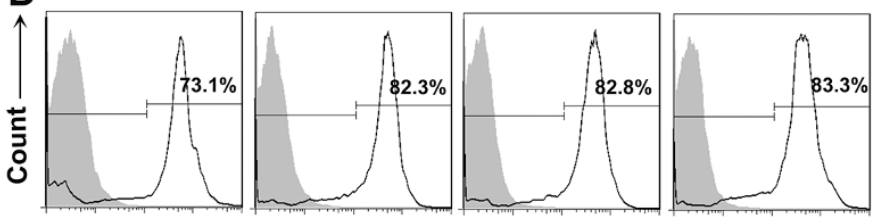

$$
\text { EpCAM }
$$

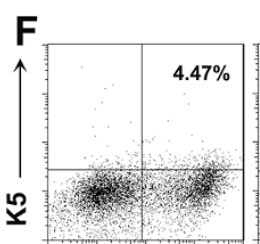

K8
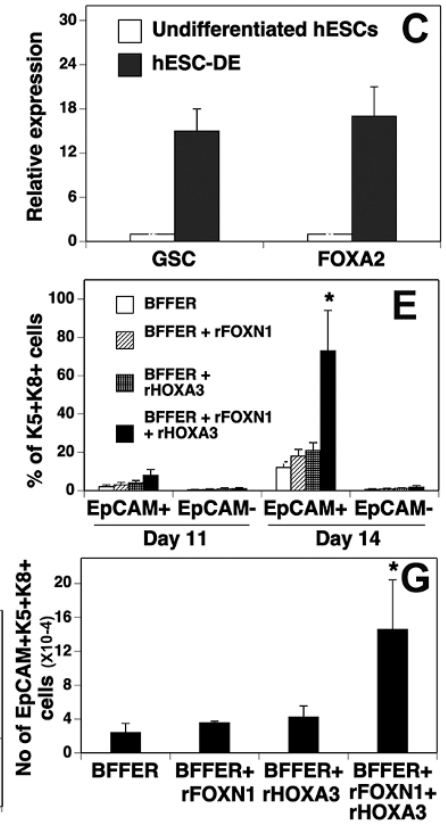

EpCAM+ gated

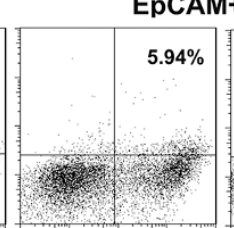

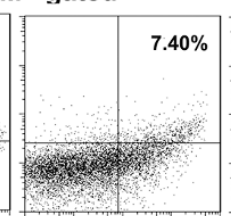

$7.40 \%$

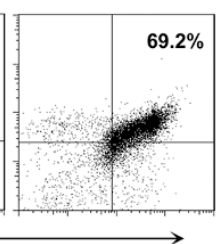

Figure $1 \mid$ Generation of TEPs from hESCs in vitro. (A) Schematic of the differentiation protocol. hESCs were induced to generate DE that further developed into TEPs in the presence of BMP4 + FGF7 + FGF10 + EGF + RA (BFFER), rFOXN1 (100 ng/ml) and rHOXA3 (200 ng/ml). The protein and/or genes that were examined for the expression are also shown. (B) Flow cytometric analysis of the expression of SOX17 at day 0-14 hESC-derived cells. The gray filled lines are isotype controls. (C) qRT-PCR analysis of the expression of GSC and FOXA2 by hESC-DE. Data are presented as relative levels of expression in hESC-derived cells versus undifferentiated hESCs. (D, E) Representative flow cytometric profiles of the expression of (D) EpCAM, and (E) K5 and K8 by EpCAM ${ }^{+}$cells by days $0-14$ hESC-derived cells that had been cultured with BFFER, rFOXN1 and rHOXA3. (F) Percentage of cells that co-expressed $\mathrm{K} 5$ and $\mathrm{K} 8$ at day 11 and $14 \mathrm{hESC}$-derived EpCAM ${ }^{+}$and EpCAM ${ }^{-}$cells. (G) The number of EpCAM ${ }^{+} \mathrm{K}^{+} \mathrm{K}^{+}$putative TEPs in day 14 hESC-derived cells that had been cultured with BFFER, rFOXN1 and/or rHOXA3. ${ }^{*} \mathrm{p}<0.05$ compared with the culture containing BFFER only. (H) Kinetics analysis of the expression of FOXN1, HOXA3, TBX1, PAX9, EYA1, and PAX1 in the hESC-derived cells from cultures containing BFFER + rFOXN1 + rHOXA3 by qRT-PCR. Data are presented as relative levels of expression on days 8,11 and 14 hESC-derived cells versus day 4 hESC-DE. hFT was used as a positive control. The data are Mean \pm SD from 3 independent experiments.

induced the differentiation of hESCs into definitive endoderm (DE) by the Human Pluripotent cell-derived Endoderm Differentiation Kit (R\&D Systems) that contains specially formulated media supplements and growth factors including Activin A and Wnt-3a. Four days later, the cells were analyzed for the expression of the DE markers SOX17, goosecoid (GSC), and FOXA2. Flow cytometric analysis showed that more than $72 \%$ of the hESC-derived cells expressed SOX17 (Figure 1B). Real-time quantitative RT-PCR (qRT-PCR) analysis showed that the expression of the GSC and FOXA2 genes was significantly increased as compared to undifferentiated hESCs (Figure 1C). These results indicate that the $\mathrm{DE}$ has been generated from hESCs.

We then directed the differentiation of the hESC-DE into TEPs. We have previously reported that the combination of fibroblast grown factor (FGF) 7, FGF10, Epithelial growth factor (EGF), and bone morphogenetic protein 4 (BMP-4) induces the differentiation of mouse ESCs into TEPs ${ }^{11,12}$. We added these growth factors to the hESC cultures. Because it has been reported that retinoic acid (RA) can enhance the development of TEPs from $\mathrm{hESCs}^{13,14}$, we also added RA. It is well known that FOXN1 is a pivotal regulator of thymic epithelium development and identity ${ }^{15,17-19}$. We have cloned and expressed recombinant (r) FOXN1 protein fused with the HIV transactivator of transcription (TAT) protein transduction domain (PTD) (amino acids 47-57) (Song Y et al. submitted for publication). It has been reported that TAT PTD mediated protein transduction with high efficiency in hESCs $^{20}$. We have shown that rFOXN1 protein, when added into culture medium, can translocate from the cell surface into the cytoplasm and nucleus (Song Y et al. submitted for publication). Some of the cultures also received rFOXN1 (50$500 \mathrm{ng} / \mathrm{ml}$ ). HOXA3 has been proposed to be the earliest regulator for thymus organogenesis ${ }^{17}$. It has been shown that the third $\alpha$ helix of the homeodomain can direct internalization of HOXA3 protein via receptor-independent passive translocation into cells ${ }^{21,22}$. We cloned and expressed the HOXA3 gene to produce a rHOXA3 protein that was confirmed by Western blot (see Supplemental Fig. S1 online). Some of the cultures additionally received rHOXA3 (100$500 \mathrm{ng} / \mathrm{ml})$.

We analyzed for the expression of EpCAM because it has been shown to be expressed by TEPs ${ }^{23}$. We found that $69-88 \%$ of day $0-14$ hESC-derived cells that had been cultured with or without rFOXN1 and/or rHOXA3 expressed EpCAM, and the percentages of $\mathrm{EpCAM}^{+}$cells did not significantly differ among groups (Figure 1D and data not shown). Studies have shown that K5 and $\mathrm{K} 8$ double positive $\left(\mathrm{K}^{+} \mathrm{K}^{+}\right)$cells contain or represent TEPs ${ }^{24-28}$. We then examined the expression of $\mathrm{K} 5$ and $\mathrm{K} 8$ from the hESCderived cells. As shown in Figure $1 \mathrm{E}$ and $\mathrm{F}$, the addition of rFOXN1 and/or rHOXA3 slightly increased the percentage of $\mathrm{K}^{+} \mathrm{K}^{+}$cells in day $11 \mathrm{hESC}$-EpCAM ${ }^{+}$cells, as did the addition 
of rFOXN1or rHOXA3 in day 14 hESC-EpCAM ${ }^{+}$cells. However, the differences did not achieve statistical significance. In contrast, the addition of rFOXN1 and rHOXA3 resulted in a significant 5.8-6.5fold increase in the percentage and number of $\mathrm{K}^{+} \mathrm{K}^{+}$cells in day 14 hESC-EpCAM ${ }^{+}$cells (Figure $1 \mathrm{E}-\mathrm{G}$ ), as compared to cultures without $r F O X N 1$ and rHOXA3. The results indicate that the combination of rFOXN1 and rHOXA3 can enhance the generation of hESC-TEPs. Because the greatest number of $\mathrm{EpCAM}^{+} \mathrm{K}^{+} \mathrm{K}^{+}$cells were generated when $\mathrm{rFOXN} 1$ and rHOXA3 were added at the concentrations of $100 \mathrm{ng} / \mathrm{ml}$ and $200 \mathrm{ng} / \mathrm{ml}$, respectively (data not shown), we used these doses in the follow-up studies. In all of the culture conditions, few hESC-EpCAM ${ }^{-}$cells were $\mathrm{K}^{+} \mathrm{K}^{+}$cells (data not shown), indicating that hESC-TEPs were located in the EpCAM ${ }^{+}$cells.

We also analyzed for the expression of the third pharyngeal pouch endoderm (PPE) and TEP related genes FOXN1, HOXA3, TBX1, $P A X 9, E Y A 1$, and PAX1 by qRT-PCR. A significantly enhanced expression of these genes in the hESC-derived cells was observed on day 11, and the expression levels of these genes in day 11 hESC-derived cells were comparable to those in day $14 \mathrm{hESC}$-derived cells (Figure $1 \mathrm{H}$ ). The expression of these molecules was confirmed at the protein level (see Supplemental Fig. S2 online, and data not shown). This is in contrast to the observation that the most $\mathrm{EpCAM}^{+} \mathrm{K}^{+} \mathrm{K}^{+}$cells were generated only after day 14 (Figure 1F, G). These results suggest that the third PPE was probably generated in our culture conditions on days 9-11, and the third PPE further developed into TEPs on day 14.

hESC-derived TEPs develop into TECs in vivo. To determine whether hESC-derived TEPs can develop into TECs in vivo, we purified hESC-derived $\mathrm{EpCAM}^{+}$and $\mathrm{EpCAM}^{-}$cells from the cultures. The cells were reaggregated in vitro and then transplanted under the kidney capsule of nude mice that had been irradiated and injected with anti-asialo-GM1 antibody to delete NK cells before the transplantation $^{29}$. Three months later, the grafts were harvested and analyzed for structure by immunofluorescence. As shown in Figure $2 \mathrm{~A}$, discrete $\mathrm{K}^{+} \mathrm{K}^{-}$cortical (green color) and $\mathrm{K} 8^{-} \mathrm{K} 5^{+}$ medullary (red color) areas were present in the $\mathrm{EpCAM}^{+}$cell graft, suggesting that hESC-TEPs could generate cTECs and mTECs and form a thymic architecture in vivo. Some of the cells were $\mathrm{K}^{+} \mathrm{K}^{+}$
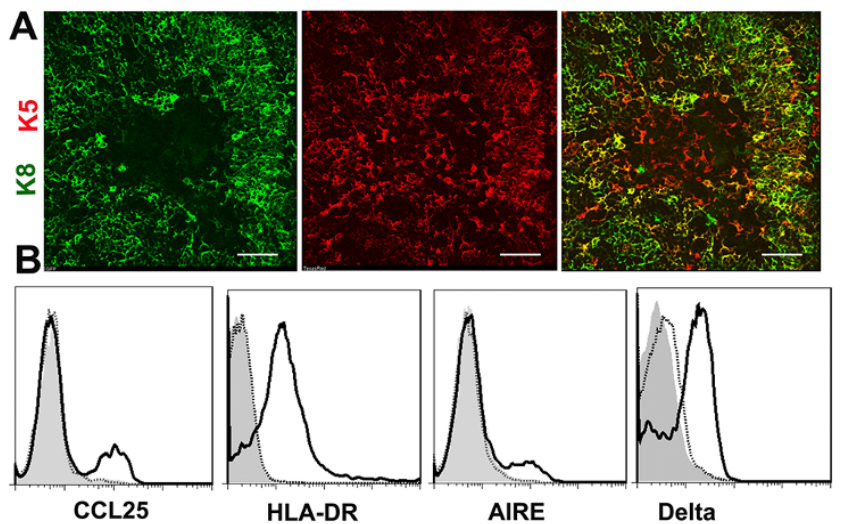

Figure $2 \mid$ hESC-TEPs develop into TECs and form a thymic architecture in vivo. $\mathrm{EpCAM}^{+}$and $\mathrm{EpCAM}^{-}$cells were purified from day $14 \mathrm{hESC}$ cultures, cell reaggregated in vitro and then transplanted under the kidney capsule of nude mice that had been irradiated ( $3 \mathrm{~Gy}$ ) and injected i.p. with $25 \mu \mathrm{l}$ anti-asialo-GM1. Twelve weeks later, the grafts were harvested. (A) Sectioned $\mathrm{EpCAM}^{+}$grafts were immunofluorescently stained using K8 and $\mathrm{K} 5$ antibodies. A representative graft is shown. Scale bar $=100 \mu \mathrm{m}$. (B) Flow cytometric analysis of the expression of CCL25, HLA-DR, AIRE, and delta molecules by $\mathrm{CD} 45^{-}$cells in the $\mathrm{EpCAM}^{+}$(solid lines) and $\mathrm{EpCAM}^{-}$(dotted lines) grafts. Isotype antibodies were used as controls (gray filled lines). (yellow color), suggesting they were residual or self-replicating TEPs. In contrast, hESC-EpCAM ${ }^{-}$cells could not form a thymic architecture in vivo (data not shown).We then analyzed the hESCTECs for the expression of molecules critical for recruiting $\mathrm{T}$ cell precursors to and/or supporting $\mathrm{T}$ cell development in the thymus. As shown in Figure 2B, CD45 ${ }^{-}$cells that contain hESC-TECs in the $\mathrm{EpCAM}^{+}$grafts expressed CCL25, HLA-DR, and AIRE molecules, whereas $\mathrm{CD}^{-} 5^{-}$cells from the $\mathrm{EpCAM}^{-}$grafts did not express these molecules. We also examined the expression of delta-like Notch ligands with an antibody that recognizes epitopes common to the human delta-like $1-4^{30}$. We found that hESC-derived TECs also expressed these ligands. Collectively, these data indicate that hESC-TEPs in vivo can further develop into TECs that express molecules critical for $\mathrm{T}$ cell development.

Transplantation of hESC-TEPs in nude mice results in long-term generation of mouse $\mathrm{T}$ cells. Since the major function of TECs is to support $\mathrm{T}$ cell development, we assessed $\mathrm{T}$ cells in the hESC$\mathrm{EpCAM}^{+}$and $\mathrm{EpCAM}^{-}$cell grafts over time. We found that CD4 and CD8 double positive (DP) and single positive (SP) T cells were generated in the hESC-EpCAM ${ }^{+}$grafts, but not in the hESCEpCAM $^{-}$grafts (Figure 3A). Importantly, $\mathrm{T}$ cell development in the $\mathrm{EpCAM}^{+}$grafts was maintained through at least week 24 (Figure 3B, C). Analysis of the expression of $\alpha \beta$ and $\gamma \delta$ TCR by $\mathrm{CD}^{+} \mathrm{T}$ cells in the EpCAM1 ${ }^{+}$grafts showed that more than $90 \%$ of the cells were $\alpha \beta$ TCR T cells (Figure 3D and Supplemental Fig. 3). $\mathrm{CD}^{+}{ }^{+} \mathrm{Foxp}^{+}{ }^{+}$regulatory $\mathrm{T}$ cells were also observed in the $\mathrm{EpCAM}^{+}$ cell-transplanted mice (Figure 3E and Supplemental Fig. 3). These results suggest that the hESC-TEPs/TECs can attract mouse $\mathrm{T}$ cell precursors to the grafts and support their development into $\mathrm{T}$ cells.

In normal animals, $\mathrm{T}$ cells usually migrate to peripheral lymphoid organs after their maturation in the thymus. We then examined the percentage and number of CD4 and CD8 T cells in the spleen. There were only a few $\mathrm{T}$ cells in the untreated or hESC-EpCAM ${ }^{-}$celltransplanted nude mice (Figure $3 \mathrm{~F}-\mathrm{H}$ ). In contrast, a considerable number of CD4 and CD8 T cells were present in the spleens of the hESC-EpCAM $^{+}$cell-transplanted nude mice 12 weeks after the transplantation (Figure $3 \mathrm{~F}$ ), suggesting $\mathrm{T}$ cells in the grafts can migrate to the periphery. A large number of CD4 and CD8 T cells, as well as $\mathrm{CD}^{+} \mathrm{Foxp}^{+}$regulatory $\mathrm{T}$ cells still existed in the spleens 24 weeks after the transplantation (Figure $3 \mathrm{G}, \mathrm{H}$ ), consistent with the data that $\mathrm{T}$ cells continued development in the grafts. Collectively, the presence of CD4 and CD8 SP T cells in the spleens and CD4 and CD8 DP and SP T cells in the grafts of the hESC$\mathrm{EpCAM}^{+}$cell-transplanted nude mice suggest that the hESC-TECs support de novo generation of $\mathrm{T}$ cells.

$\mathrm{T}$ cells in the hESC-TEP-transplanted mice are functional. Functionally mature $\mathrm{T}$ cells are characterized by their ability to proliferate and produce cytokines in response to $\mathrm{T}$ cell receptor (TCR)-mediated signals ${ }^{30}$. To determine whether peripheral T cells in the hESC-TEP-transplanted mice were functional, we first examined $\mathrm{T}$ cell proliferation following TCR stimulation. To this end, splenocytes were loaded with carboxyfluorescein diacetate succinimidyl ester (CFSE), and then stimulated with anti-CD3 and CD28 antibodies. Cell proliferative ability was assessed by the loss of CFSE. As shown in Figure $4 \mathrm{~A}$ and $4 \mathrm{~B}$, a significantly higher proportion of $\mathrm{CD}^{+}$and $\mathrm{CD}^{+} \mathrm{T}$ cells proliferated following antiCD3 and anti-CD28 stimulation in the hESC-EpCAM ${ }^{+}$celltransplanted mice than in the hESC-EpCAM ${ }^{-}$cell -transplanted or untreated nude mice. We then analyzed IL-2 producing T cells after TCR stimulation. Similarly, a significantly higher proportion of $\mathrm{CD}^{+}$and $\mathrm{CD}^{+} \mathrm{T}$ cells were able to produce IL-2 following TCR stimulation in the hESC-EpCAM ${ }^{+}$cell-transplanted mice than in the control mice (Figure 4C). Taken together, these results suggest that the newly formed $\mathrm{T}$ cells in the hESC-TEP-transplanted mice are functional. 

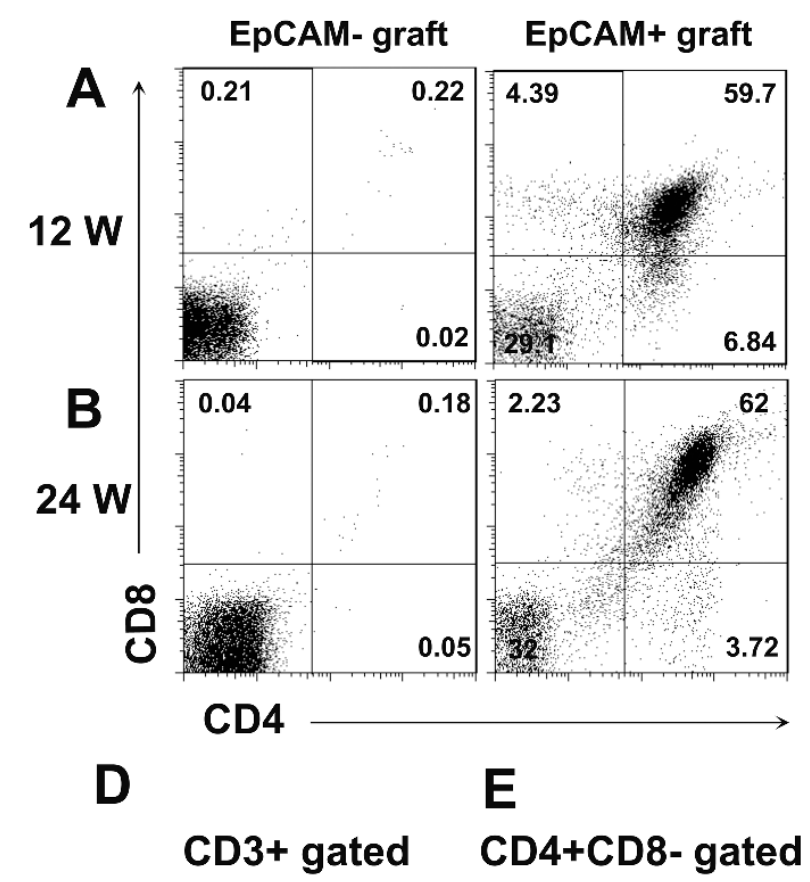

$\square$ untreated $\square$ EpCAM- $\square$ EpCAM+
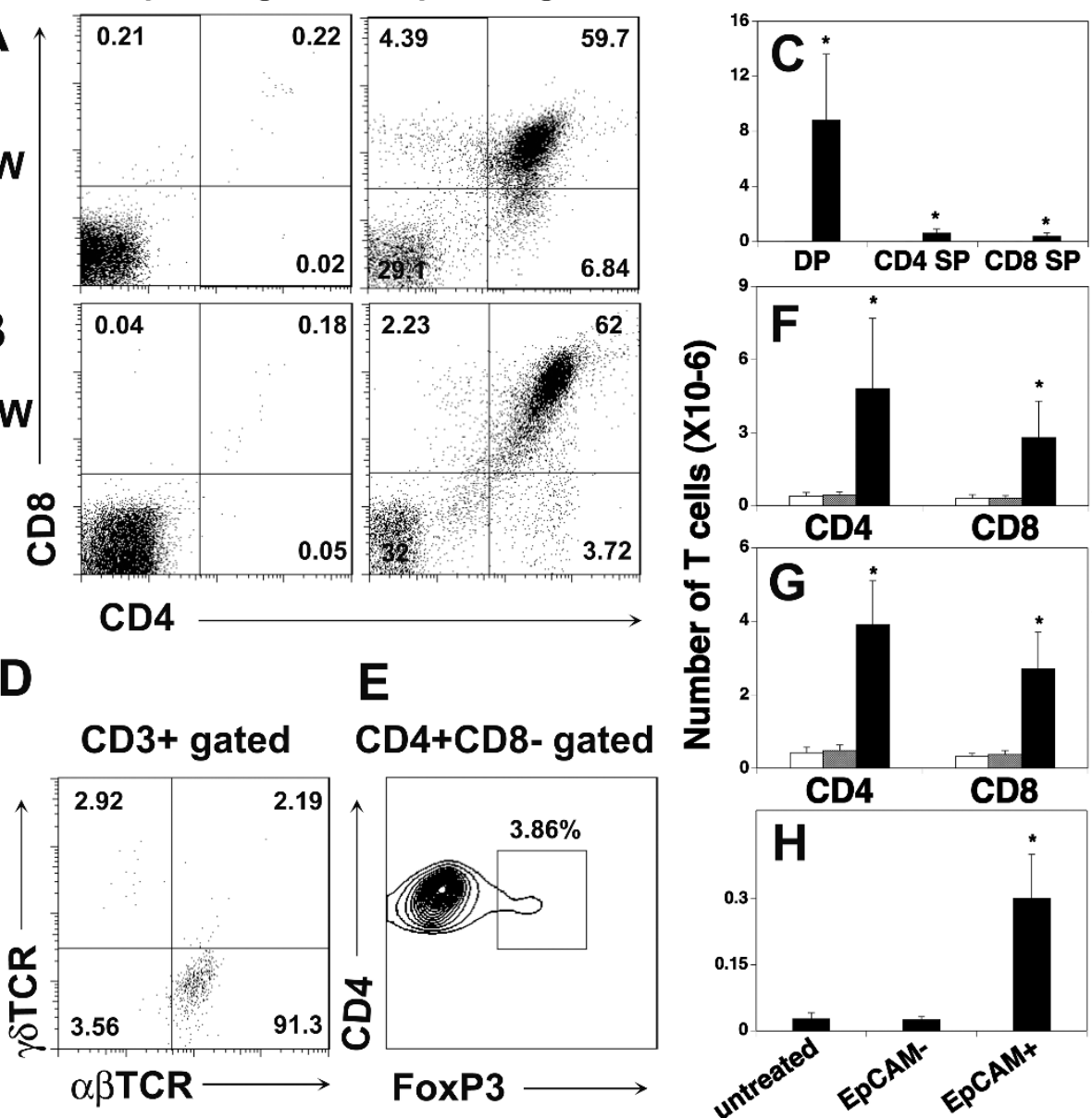

Figure 3 hESC-TECs support mouse T cell development in vivo. EpCAM ${ }^{+}$and EpCAM ${ }^{-}$cells were purified from day $14 \mathrm{hESC}$ cultures, reaggregated in vitro and then transplanted under the kidney capsule of nude mice as in Figure 2. The grafts and spleens were harvested and analyzed (A, F) 12 and (C-E, G, H) 24 weeks (w) later. (A-E) T cell development in the grafts was analyzed. Representative flow cytometric profiles of (A, B) CD4 and CD8 DP, and SP, and $(\mathrm{C})$ the number of T cells in the $\mathrm{EpCAM}^{+}$and $\mathrm{EpCAM}^{-}$cell grafts. Representative flow cytometric profiles of (D) the expression of $\alpha \beta$ and $\gamma \delta$ TCR by $\mathrm{CD}^{+} \mathrm{T}$ cells, and (E) $\mathrm{CD}^{+}{ }^{+} \mathrm{Foxp}^{+}{ }^{+}$regulatory T cells in the EpCAM ${ }^{+}$cell grafts. The number of (F, G) CD4 and CD8 T cells, as well as (H) CD4 ${ }^{+}$Foxp ${ }^{+}$ regulatory $\mathrm{T}$ cells in the spleens of the $\mathrm{EpCAM}^{+}$and $\mathrm{EpCAM}^{-}$cell-transplanted mice were analyzed by flow cytometry. Untreated nude mice were used as controls. The data are representative of 3 independent experiments with $5-6$ mice per group. ${ }^{*} \mathrm{p}<0.05$ compared with untreated or EpCAM ${ }^{-}$cell-treated nude mice.

Transplantation of hESC-TEPs and human hematopoietic precursors lead to the generation of functional human $\mathrm{T}$ cells. Having shown that hESC-TECs supported the development of functional mouse $\mathrm{T}$ cells in vivo, we wanted to determine whether they were able to support human $\mathrm{T}$ cell development. To this end, irradiated NOD.Cg-Prkdcscid Il2rgtm $1 \mathrm{Wjl} / \mathrm{SzJ}$ (NSG) mice were transplanted with hESC-derived $\mathrm{EpCAM}^{+}$cells or $\mathrm{EpCAM}^{-}$ control cells under the kidney capsule. These mice were also injected i.v. with human $\mathrm{BM} \mathrm{CD}^{+} 4^{+}$hematopoietic precursors. Twelve weeks later, the grafts, peripheral blood (PB) and spleens were examined for the presence of human $\mathrm{T}$ cells. The hESCEpCAM $^{+}$grafts contained human CD4 and CD8 DP and SP $\mathrm{T}$ cells, whereas hESC-EpCAM ${ }^{-}$grafts did not (Figure 5A, B). Analysis of $\mathrm{PB}$ mononuclear cells (PBMCs) showed that very low percentages of human CD4 and CD8 SP T cells were present in the mice that had been transplanted with hESC-EpCAM ${ }^{-}$cells and BM $\mathrm{CD}_{3} 4^{+}$hematopoietic precursors or $\mathrm{BM} \mathrm{CD} 34^{+}$hematopoietic precursors only (Figure $5 \mathrm{C}$ ), indicating that the thymus in the NSG mice supported low levels of human $\mathrm{T}$ cell development. In contrast, significantly higher percentages of human CD4 and CD8 SP $\mathrm{T}$ cells were present in the PBMCs of the hESC-EpCAM ${ }^{+}$ cell-transplanted mice (Figure 5C), consistent with the data that human $\mathrm{T}$ cell developed in the grafts. Similar results were obtained when the spleens were analyzed (data now shown).

To determine whether the human $\mathrm{T}$ cells are functional, splenic human $\mathrm{T}$ cells were examined for proliferation and cytokine-producing $\mathrm{T}$ cells after stimulation ${ }^{31}$. As shown in Figure 5D and E, a significantly higher proportion of human CD4 T cells or CD8 T cells in the hESC-EpCAM ${ }^{+}$cell-transplanted mice were capable of proliferation after TCR stimulation, as compared to unstimulated cells. Similarly, a significantly higher proportion of human CD3 T cells in the hESC-EpCAM ${ }^{+}$cell-transplanted mice were able to produce IL-2 and IFN- $\gamma$ after stimulation (Figure 5F, G). These data suggest that the human T cells were functional. Collectively, hESC-TECs in vivo can also support the development of functional human $\mathrm{T}$ cells.

\section{Discussion}

The thymus in mice initially arises from the endoderm of the third pharyngeal pouch ${ }^{16,18}$. We first induced the differentiation of hESCs into DE that was further induced to develop into TEPs. Although our protocol does not include separate steps that direct the differentiation of DE into the third PPE, or anterior foregut endoderm (AFE) 

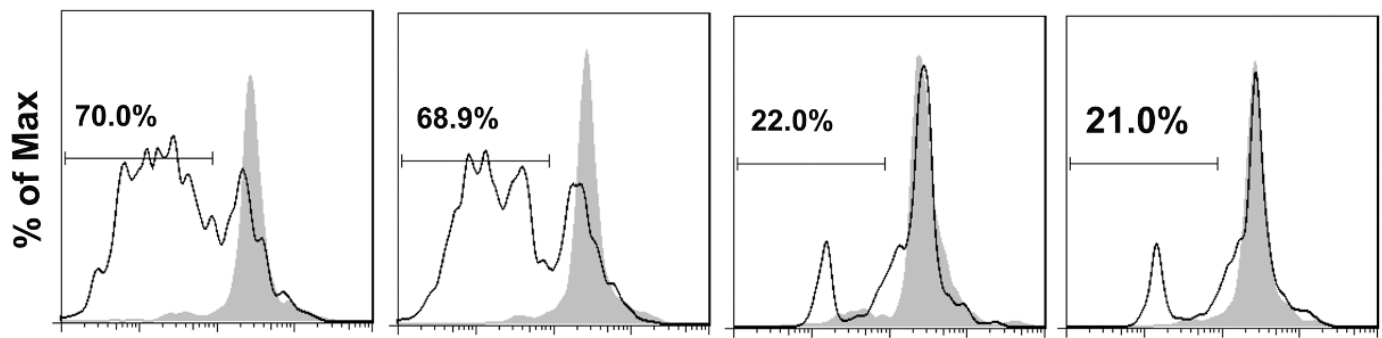

CFSE

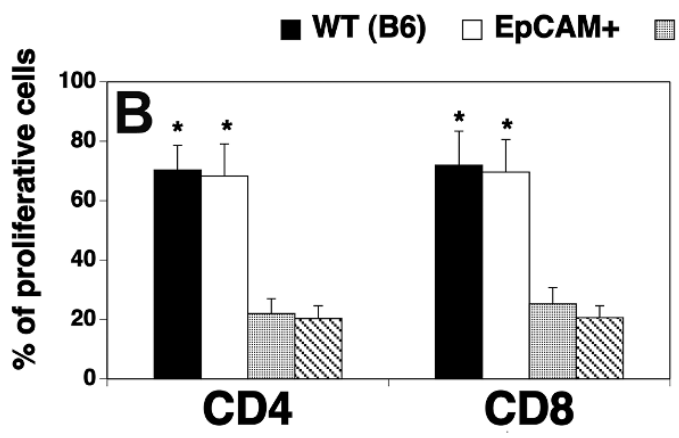

EpCAM- $⿴$ untreated nude

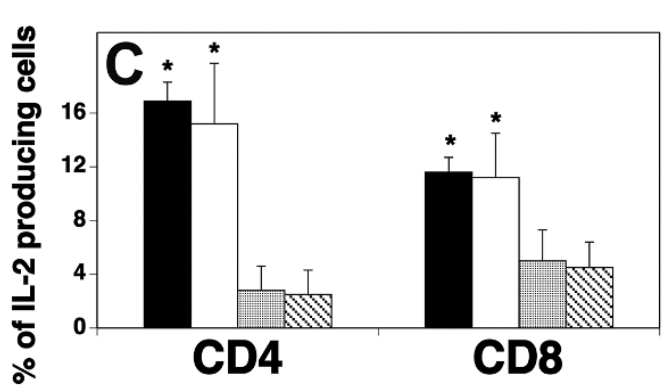

Figure $4 \mid$ Peripheral T cells in the hESC-TEP-transplanted mice are functional. hESC-derived $\mathrm{EpCAM}^{+}$and $\mathrm{EpCAM}^{-}$cell reaggregates were transplanted under the kidney capsule of nude mice as in Figure 2. Untreated nude mice were used as controls. Twelve weeks later the spleens were harvested, and splenocytes were labeled with CFSE. Splenocytes were cultured in the presence of anti-CD3 and anti-CD28 antibodies (5 $\mu \mathrm{g} / \mathrm{ml})$ for 4 days. (A, B) The cells were stained with anti-CD4 and CD8 antibodies and analyzed for CFSE levels by CD4 ${ }^{+}$and CD8 ${ }^{+} \mathrm{T}$ cells. (A) Representative flow cytometric analysis of CFSE distribution of $\mathrm{CD}^{+} \mathrm{T}$ cells (nonstimulated cells are represented by gray filled lines), and (B) statistical analysis of $\mathrm{T}$ cell proliferation is shown. (C) The cells were stained with antibodies for cell surface markers and intracellular cytokine IL-2. The percentage of IL-2 positive cells in $\mathrm{CD}^{+}$and $\mathrm{CD}^{+} \mathrm{T}$ cells was determined by flow cytometry. The data are representative of 3 independent experiments with 5-6 mice per group. $* \mathrm{p}<0.05$ compared with untreated nude mice.

and ventral pharyngeal endoderm (VPE) $)^{13,14}$, our kinetic analysis of the expression genes related to PPE and TEPs indicates that on days 9-11 the DE developed into the third PPE or VPE which then further developed into TEPs on day 14 .

It has been reported that mesenchymal-epithelial interaction is important for early stages of thymic organogenesis ${ }^{1,2,32}$. The interaction is medicated, at least in part, by FGF7 and FGF10 produced by mesenchymal cells ${ }^{1,2,33}$. The BMP4 signaling pathway is also involved in the initial patterning of the thymus ${ }^{16}$. Therefore, we have included FGF7, FGF10, and BMP4 in our cultures for TEP differentiation. However, our data show that these factors are not sufficient to direct the differentiation of hESC-DE into TEPs, and that HOXA3 and FOXN1 are also required for the differentiation.

HOXA3, a member of the HOX family of transcription factors, has been proposed to be the earliest regulator for thymus organogenesis $^{17,34}$. Mice homozygous for HOXA3 deletion are athymic ${ }^{34}$. FOXN1 is a member of the winged helix/forkhead box transcription factor family. A loss-of-function mutation in FOXN1 in mice, rats and humans results in the display the 'nude' phenotype, which is characterized by congenital athymia and hairlessness ${ }^{35}$. Many studies have shown that FOXN1 plays a critical role in TEC development ${ }^{17-19,36}$. It has been reported that the thymic rudiment in nude mice resembles respiratory epithelium, suggesting that FOXN1 may also be involved in the specification of a thymic developmental fate of the $3^{\text {rd }} \mathrm{PPE}^{37}$. Because both the FOXN1 and HOXA3 genes in human and mouse are highly conserved in sequence and function ${ }^{17,19,38,39}$, we have used the murine forms of rFOXN1 and rHOXA3 in our studies. It was anticipated they would be functional in human cells. Indeed, our data showed that the addition of these two molecules significantly enhanced the generation of hESC-TEPs. The role of HOXA3 and FOXN1 in hESC-TEP generation remains to be further defined.
It is possible that HOXA3 and FOXN1 induce the differentiation of hESC-DE into TEPs and/or promote the survival of hESC-TEPs.

Despite the addition of rFOXN1 protein to our culture system and induced endogenous expression of FOXN1, the hESC-TEPs could not spontaneously differentiate into TECs in vitro. However, when transplanted in vivo, the hESC-TEPs were able to further develop into TECs. It has been shown that, in addition to the intrinsic factor FOXN1, extrinsic signals from developing $\mathrm{T}$ cells are also important for TEC development $t^{2,7,25,40-42}$. It is possible that hESC-TEPs that express the chemokine CCL25 and other chemokines attract T cell precursors from the blood. TEPs interact with the $\mathrm{T}$ cell precursors to develop into TECs that, in turn, support the development of T cells from the T cell precursors.

In summary, we have developed a new protocol to efficiently generate TEPs from hESCs in vitro. Transplantation of purified hESC-TEPs results in the development of hESC-TECs that support long-term development of mouse $\mathrm{T}$ cells and a higher level of engraftment of human T cells. Our studies have important implications for the treatment of patients with primary and secondary T cell immunodeficiency.

\section{Methods}

Mice. NU/J nude and NSG mice were purchased from Jackson Laboratory. Mice were housed, treated, and handled according to protocols approved by the University of Connecticut Animal Care and Use Committee.

Molecular cloning and expression of rHoxa3 protein. HOXA3-mCherry containing pSecTag2A vector was kindly provided by Dr. Kimberly A. Mace in the University of Manchester ${ }^{22}$. To obtain rHOXA3 without mCherry, the Hoxa3 gene was amplified from the vector using primers $5^{\prime}$ -

GGTACCGAGCTCGGATCCAATGCAAAAAGCGACCTACTAC- ${ }^{\prime}$ and

5' - GAGTTTTTGTTCGGGCCCCAGGTGGGTGAGCTTGGGCG-3'. The PCR product was cloned into the pSecTag2A vector (Invitrogen, Carlsbad, CA) using the 

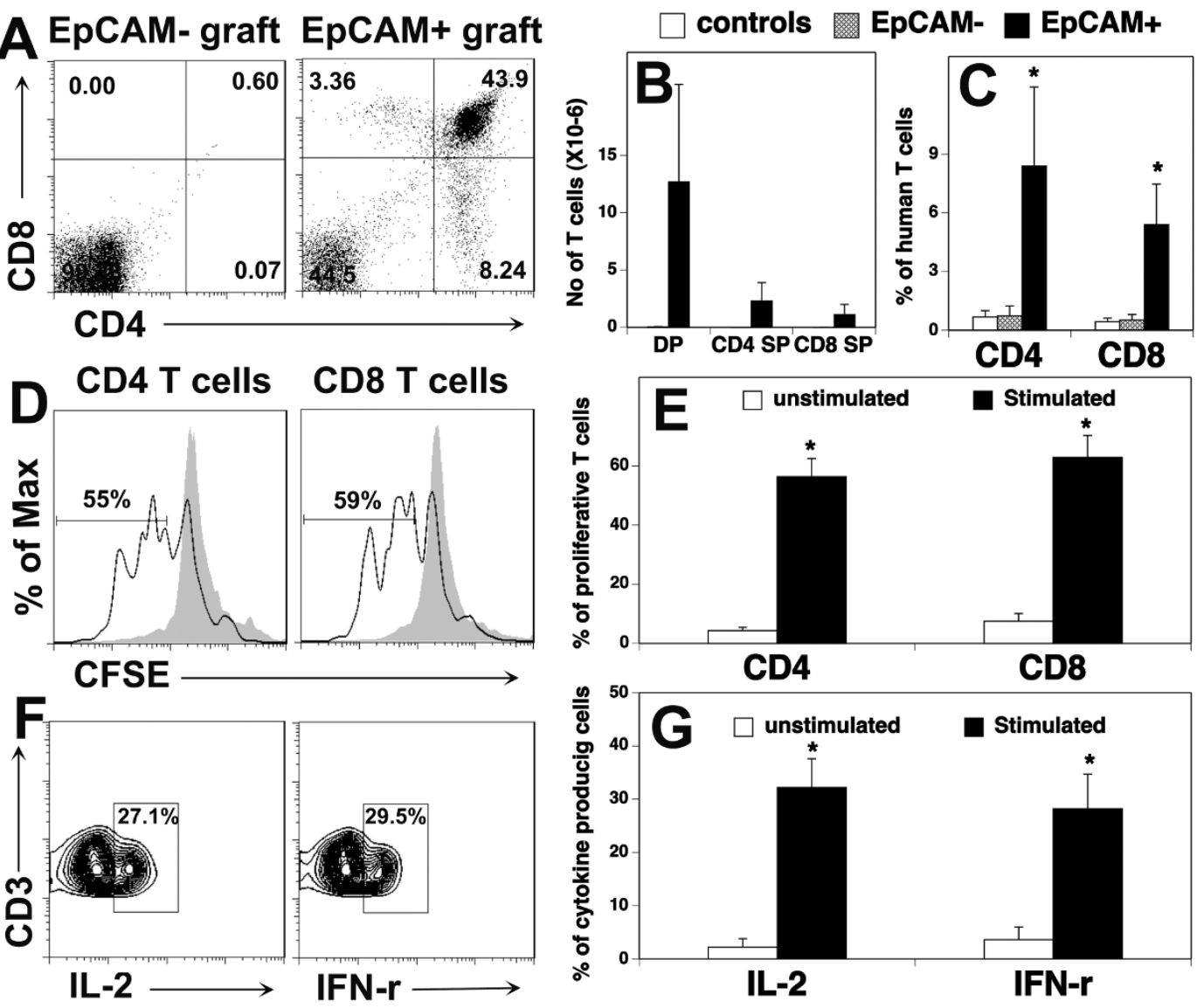

Figure $5 \mid$ hESC-TECs support the development of functional human T cells. Four to six-week-old NSG mice were irradiated (2 Gy), transplanted under the kidney capsule with $\mathrm{EpCAM}^{+}$or $\mathrm{EpCAM}^{-}$cell reaggregates, and injected i.v. with human $\mathrm{BM} \mathrm{CD}^{+} 4^{+}$cells. Mice that had been injected i.v. with human $\mathrm{BM} \mathrm{CD} 34^{+}$cells only were used as a control. Twelve weeks later, the grafts, blood and spleens were harvested and analyzed. (A) Representative flow cytometric analysis of human CD4 and CD8 DP, and SP T cells, (B) the number of DP, CD4 SP and CD8 SP T cells in the grafts. (C) The percentage of human CD4 and CD8 T cells in the PBMCs. (D, E) Splenocytes from the EpCAM ${ }^{+}$cell-transplanted mice were labeled with CFSE and cultured in the presence of Dynabeads ${ }^{\circledR}$ human T-Activator CD3/CD28 for 3 days. The cells were then stained with anti-huCD4 and huCD8 antibodies and analyzed for CFSE levels by CD4 ${ }^{+}$and $\mathrm{CD} 8^{+} \mathrm{T}$ cells. (D) Representative flow cytometric analysis of CFSE distribution of CD4 ${ }^{+}$or CD8 ${ }^{+} \mathrm{T}$ cells (unstimulated cells are represented by gray filled lines), (E) the percentage of proliferated T cells. (F, G) The splenocytes were cultured in the presence or absence of PMA and ionomycin. The cells were then stained with anti-huCD3 and human IL-2 or IFN- $\gamma$ antibodies and analyzed by flow cytometry. (F) Representative flow cytometric profiles, and $(\mathrm{G})$ the percentage of IL- 2 or IFN- $\gamma$ producing $\mathrm{CD}^{+} \mathrm{T}$ cells. The data are representative of 3 independent experiments with 5 mice per group. ${ }^{*} \mathrm{p}<0.05$ compared with control or unstimulated group.

In-Fusion HD Cloning Kit (Clontech Laboratories, Mountain View, CA) according to the manufacture's instruction. The pSecTag2A vector containing the Hoxa3 gene was confirmed by DNA sequencing, and then transfected into CHO-S cells (Invitrogen). rHOXA3 or rHOXA3-mCherry was collected from the supernatant of the CHO-S cells that had been transfected with the pSecTag2A vector containing the Hoxa3 or Hoxa3-mCherry gene, and verified by Western blot using a HOXA3 antibody. We used equimolar amounts of rHOXA3 and rHOXA3-mCherry in our initial studies, and obtained similar results (data not shown).

Cell culture. Undifferentiated hESCs (H9 and CT2) were maintained on mouse embryo fibroblast feeder layers (GlobalStem, Gaithersburg, MD) in DMEM/F12 supplemented with $20 \%$ Knockout serum replacement (Invitrogen), $1 \mathrm{mM}$ nonessential amino acids, Glutamax, penicillin/ streptomycin, $0.55 \mathrm{mM} 2$ mercaptoethanol (Invitrogen) and $4 \mathrm{ng} / \mathrm{ml}$ recombinant human FGF2 (R\&D Systems, Minneapolis, MN). For the differentiation of hESCs into DE, the cells $\left(4 \times 10^{3}\right.$ cells/well) were cultured in the medium from the Human Pluripotent cellderived Endoderm Differentiation Kit (R\&D Systems) according to the manufacturer's instructions for 4 days. For the differentiation of hESC-DE into TEPs, the cells were cultured in the presence of human FGF7 $(20 \mathrm{ng} / \mathrm{ml})$, FGF10 (20 ng/ml), EGF (50 ng/ml), BMP-4 (5 ng/ml) (R\&D Systems or Pepro Tech, Rocky Hill, NJ), 0.5 $\mu$ M all-trans RA (Sigma-Aldrich, St. Louis, MO), rFOXN1 $(100 \mathrm{ng} / \mathrm{ml})$ and $\mathrm{rHOXA} 3$ protein $(200 \mathrm{ng} / \mathrm{ml})$,

Flow cytometry analysis. Cells were stained with the fluorochrome-conjugated antibodies as described ${ }^{43,44}$. For intracellular staining, the cells were first permeabilized with a BD Cytofix/Cytoperm solution for 20 minutes at $4{ }^{\circ} \mathrm{C}$. Direct or indirect staining of fluorochrome-conjugated antibodies included: mouse CD4, mouse CD8, mouse T cell receptor (TCR) $\beta$, TCR $\gamma \delta$, FoxP3, mouse or human (hu) interleukin-2 (IL-2), huCD4, CD8, huCD45, huCD3, huEpCAM, interferon (IFN)- $\gamma$, and HLA-DR (BioLegend or BD Biosciences, San Diego, CA), k5, Delta, and CCL25 (Santa Cruz Biotechnology, Santa Cruz, CA), k8 (US Biological, Swampscott, MA), AIRE, SOX17 (Millipore, Billerica, MA), fluorescein isothiocyanate (FITC), or phycoerythrin (PE) labeled anti-rat, or rabbit IgG (BD Biosciences). The samples were analyzed on a FACSCalibur (Becton and Dickinson Company). Data analysis was performed using FlowJo software (Ashland, OR).

qRT-PCR. Total RNA from cells was isolated from cells, and cDNA was synthesized as described ${ }^{45}$. qRT- PCR was performed with the Power SYBR green mastermix (Applied Biosystems, UK) using the 7500 real-time PCR system (Applied Biosystems, $\mathrm{UK})$. After normalization to $G A P D H$, samples were plotted relative to undifferentiated hESCs or day 4 hESC-derived DE. Human fetal thymus (HFT, obtained from Advanced Bioscience Resource) was used as a positive control. Primers are summarized in Supplemental Table 1.

Western blot. Proteins were loaded on a 10\% SDS-PAGE, transferred to a polyvinylidene fluoride membrane, and then incubated with following antibodies: anti-HOXA3 (Abcam, Cambridge, MA), FOXN1 (Thermo Scientific, or Fitzgerald, Acton, MA), PAX-1, or PAX-9 (Santa Cruz Biotechnology) ${ }^{46,47}$, and HRP conjugated secondary antibody. The membrane with proteins and antibodies was developed with Super Signal ${ }^{\circledR}$ West Pico chemiluminescent Substrate (Thermo Scientific).

Immunomagnetic cell separation. Single-cell suspensions from ESC-derived cells were stained with PE labelled anti-huEpCAM antibody, washed, and stained with anti-PE MicroBeads (Miltenyi Biotec, Auburn, CA). EpCAM ${ }^{+}$and EpCAM ${ }^{-}$cells 
were selected using a magnetic-activated cell sorter immunomagnetic separation system (Miltenyi Biotec).

Kidney capsule grafting and bone marrow transplantation. Purified hESC-derived $\mathrm{EpCAM}^{+}$or EpCAM ${ }^{-}$cells $\left(1-2 \times 10^{6}\right)$ were subjected to reaggregate cultures for 24-48 hours, as described ${ }^{12}$. Four to six-week-old NU/J nude and NSG mice were used as recipients. The NU/J nude mice were injected i.p. with $25 \mu \mathrm{l}$ of anti-asialoGM1 (eBioscience, San Diego, CA) on day $-1^{29}$, irradiated (3 Gy) and transplanted under the kidney capsule with the solidified reaggregate of the hESC-derived cells on day 0. NSG mice were irradiated ( 2 Gy), transplanted under the kidney capsule with the solidified reaggregate of the hESC-derived cells, and injected i.v. with human bone marrow $\mathrm{CD}_{3} 4^{+}$cells $\left(1 \times 10^{5}\right.$ cells/mouse, obtained from AllCells, LLC, Alameda, CA). Twelve to 24 weeks after implantation, the grafts, spleens and blood were harvested and analyzed.

Immunohistology and confocal microscopy. Immunohistological analysis of grafted thymus tissues was performed according to a modified protocol ${ }^{12}$. Briefly, tissues were incubated in $4 \%$ paraformaldeyde for 4 hours followed by incubation in $30 \%$ sucrose solution overnight. The tissues were embedded in OCT medium, snap frozen, and subsequently cut into 5 micrometer sections. The sections were stained with anti K8 monoclonal antibody (clone: CAM5.2, BD Biosciences) and rabbit anti K5 antibody (Abcam, Cambridge, MA), followed by FITC-conjugated anti-mouse IgG (Sigma) and AlexaFluor-546-conjugated goat anti-rabbit IgG (Invitrogen). The cells were observed under a Nikon A1R confocal microscope (Nikon, Kanagawa, Japan).

T cell proliferation assays. Splenocytes were stained with $5 \mu \mathrm{M}$ CFSE (Invitrogen) for $15 \mathrm{~min}$. at $37^{\circ} \mathrm{C}$. The cells were then cultured in a 96 -well plate $\left(4 \times 10^{5}\right.$ cells/well $)$ in the presence of $5 \mu \mathrm{g} / \mathrm{ml}$ of plate-bound anti-mouse CD3 and anti-mouse CD28 antibodies or Dynabeads ${ }^{\circledR}$ human T-Activator CD3/CD28 (Invitrogen) for 3 days according the manufacture's instructions. The cells were then stained with anti-CD4 and CD8 antibodies and analyzed for CFSE levels by flow cytometry.

Statistical analysis. P-values were based on the two-sided Student's T test. A confidence level above $95 \%(p<0.05)$ was determined to be significant.

1. Anderson, G., Lane, P. J. \& Jenkinson, E. J. Generating intrathymic microenvironments to establish T-cell tolerance. Nat Rev Immunol 7, 954-963 (2007).

2. Chidgey, A., Dudakov, J., Seach, N. \& Boyd, R. Impact of niche aging on thymic regeneration and immune reconstitution. Semin Immunol 19, 331-340 (2007).

3. Ciofani, M. \& Zuniga-Pflucker, J. C. The thymus as an inductive site for T lymphopoiesis. Annu Rev Cell Dev Biol 23, 463-493 (2007).

4. Jenkinson, W. E., Bacon, A., White, A. J., Anderson, G. \& Jenkinson, E. J. An epithelial progenitor pool regulates thymus growth. Journal of immunology (Baltimore, $M d$ : 1950) 181, 6101-6108 (2008).

5. Lynch, H. E. et al. Thymic involution and immune reconstitution. Trends Immunol 30, 366-373 (2009).

6. Taub, D. D. \& Longo, D. L. Insights into thymic aging and regeneration. Immuno Rev 205, 72-93 (2005)

7. Zediak, V. P. \& Bhandoola, A. Aging and T cell development: interplay between progenitors and their environment. Semin Immunol 17, 337-346 (2005)

8. Rossi, S. W. et al. Keratinocyte growth factor (KGF) enhances postnatal T-cell development via enhancements in proliferation and function of thymic epithelial cells. Blood 109, 3803-3811 (2007).

9. van den Brink, M. R., Alpdogan, O. \& Boyd, R. L. Strategies to enhance T-cell reconstitution in immunocompromised patients. Nat Rev Immunol 4, 856-867 (2004).

10. Williams, K. M., Hakim, F. T. \& Gress, R. E. T cell immune reconstitution following lymphodepletion. Semin Immunol 19, 318-330 (2007).

11. Lai, L. et al. Mouse embryonic stem cell-derived thymic epithelial cell progenitors enhance $\mathrm{T}$-cell reconstitution after allogeneic bone marrow transplantation. Blood 118, 3410-3418 (2011).

12. Lai, L. \& Jin, J. Generation of thymic epithelial cell progenitors by mouse embryonic stem cells. Stem Cells 27, 3012-3020 (2009).

13. Parent, A. V. et al. Generation of functional thymic epithelium from human embryonic stem cells that supports host $\mathrm{T}$ cell development. Cell stem cell 13, 219-229 (2013).

14. Sun, X. et al. Directed Differentiation of Human Embryonic Stem Cells into Thymic Epithelial Progenitor-like Cells Reconstitutes the Thymic Microenvironment In Vivo. Cell stem cell 13, 230-236 (2013).

15. Soh, C. L. et al. FOXN1 (GFP/w) Reporter hESCs Enable Identification of Integrin-beta4, HLA-DR, and EpCAM as Markers of Human PSC-Derived FOXN1(+) Thymic Epithelial Progenitors. Stem cell reports 2, 925-937 (2014).

16. Gordon, J. \& Manley, N. R. Mechanisms of thymus organogenesis and morphogenesis. Development 138, 3865-3878 (2011).

17. Manley, N. R. \& Condie, B. G. Transcriptional regulation of thymus organogenesis and thymic epithelial cell differentiation. Progress in molecular biology and translational science 92, 103-120 (2010)

18. Rodewald, H. R. Thymus organogenesis. Annu Rev Immunol 26, 355-388 (2008).
19. Zhang, Z., Burnley, P., Coder, B. \& Su, D. M. Insights on FoxN1 biological significance and usages of the "nude" mouse in studies of T-lymphopoiesis. International journal of biological sciences 8, 1156-1167 (2012).

20. Kwon, Y. D. et al. Cellular manipulation of human embryonic stem cells by TAT PDX1 protein transduction. Mol Ther 12, 28-32 (2005).

21. Derossi, D. et al. Cell internalization of the third helix of the Antennapedia homeodomain is receptor-independent. J Biol Chem 271, 18188-18193 (1996).

22. Mahdipour, E., Charnock, J. C. \& Mace, K. A. Hoxa3 promotes the differentiation of hematopoietic progenitor cells into proangiogenic Gr-1 $+\mathrm{CD} 11 \mathrm{~b}+$ myeloid cells. Blood 117, 815-826 (2011).

23. Rossi, S. W., Jenkinson, W. E., Anderson, G. \& Jenkinson, E. J. Clonal analysis reveals a common progenitor for thymic cortical and medullary epithelium. Nature 441, 988-991 (2006).

24. Klug, D. B. et al. Interdependence of cortical thymic epithelial cell differentiation and T-lineage commitment. Proceedings of the National Academy of Sciences of the United States of America 95, 11822-11827 (1998).

25. Klug, D. B., Carter, C., Gimenez-Conti, I. B. \& Richie, E. R. Cutting edge: thymocyte-independent and thymocyte-dependent phases of epithelial patterning in the fetal thymus. Journal of immunology (Baltimore, Md: 1950) 169, 2842-2845 (2002).

26. Osada, M. et al. DKK1 mediated inhibition of Wnt signaling in postnatal mice leads to loss of TEC progenitors and thymic degeneration. PloS one 5, e9062 (2010).

27. Popa, I. et al. Regeneration of the adult thymus is preceded by the expansion of $\mathrm{K} 5+\mathrm{K} 8+$ epithelial cell progenitors and by increased expression of Trp63, cMyc and Tcf3 transcription factors in the thymic stroma. Int Immunol 19, 1249-1260 (2007).

28. Bleul, C. C. et al. Formation of a functional thymus initiated by a postnatal epithelial progenitor cell. Nature 441, 992-996 (2006).

29. Khan, A. et al. Discordant xenogeneic neonatal thymic transplantation can induce donor-specific tolerance. Transplantation 63, 124-131 (1997).

30. Clark, R. A., Yamanaka, K., Bai, M., Dowgiert, R. \& Kupper, T. S. Human skin cells support thymus-independent T cell development. J Clin Invest 115, 3239-3249 (2005).

31. Awong, G. et al. Human proT-cells generated in vitro facilitate hematopoietic stem cell-derived T-lymphopoiesis in vivo and restore thymic architecture. Blood 122, 4210-4219 (2013)

32. Gill, J., Malin, M., Hollander, G. A. \& Boyd, R. Generation of a complete thymic microenvironment by MTS24(+) thymic epithelial cells. Nat Immunol 3, 635-642 (2002)

33. Jenkinson, W. E., Rossi, S. W., Parnell, S. M., Jenkinson, E. J. \& Anderson, G PDGFRalpha-expressing mesenchyme regulates thymus growth and the availability of intrathymic niches. Blood 109, 954-960 (2007).

34. Manley, N. R. \& Capecchi, M. R. The role of Hoxa-3 in mouse thymus and thyroid development. Development 121, 1989-2003 (1995).

35. Palamaro, L. et al. FOXN1 in organ development and human diseases. International reviews of immunology 33, 83-93 (2014).

36. Chen, L., Xiao, S. \& Manley, N. R. Foxn1 is required to maintain the postnatal thymic microenvironment in a dosage-sensitive manner. Blood 113, 567-574 (2009).

37. Dooley, J., Erickson, M., Roelink, H. \& Farr, A. G. Nude thymic rudiment lacking functional foxn1 resembles respiratory epithelium. Dev Dyn 233, 1605-1612 (2005).

38. Nehls, M., Pfeifer, D., Schorpp, M., Hedrich, H. \& Boehm, T. New member of the winged-helix protein family disrupted in mouse and rat nude mutations. Nature 372, 103-107 (1994).

39. Schorpp, M., Hofmann, M., Dear, T. N. \& Boehm, T. Characterization of mouse and human nude genes. Immunogenetics 46, 509-515 (1997).

40. Anderson, G. et al. Establishment and functioning of intrathymic microenvironments. Immunol Rev 209, 10-27 (2006).

41. Hollander, G. A. et al. Developmental control point in induction of thymic cortex regulated by a subpopulation of prothymocytes. Nature 373, 350-353 (1995).

42. van Ewijk, W., Shores, E. W. \& Singer, A. Crosstalk in the mouse thymus. Immunol Today 15, 214-217 (1994).

43. Jin, J., Goldschneider, I. \& Lai, L. In vivo administration of the recombinant IL-7/ hepatocyte growth factor beta hybrid cytokine efficiently restores thymopoiesis and naive $\mathrm{T}$ cell generation in lethally irradiated mice after syngeneic bone marrow transplantation. Journal of immunology (Baltimore, Md : 1950) 186, 1915-1922 (2011).

44. Lai, L., Zhang, M., Song, Y. \& Rood, D. Recombinant IL-7/HGFbeta Hybrid Cytokine Enhances T Cell Recovery in Mice Following Allogeneic Bone Marrow Transplantation. PloS one 8, e82998 (2013).

45. Yan, Y. et al. Tbx1 modulates endodermal and mesodermal differentiation from mouse induced pluripotent stem cells. Stem cells and development 23, 1491-1500 (2014).

46. Schnittger, S. et al. Paxl, a member of the paired box-containing class of developmental control genes, is mapped to human chromosome 20p11.2 by in situ hybridization (ISH and FISH). Genomics 14, 740-744 (1992).

47. Stapleton, P., Weith, A., Urbanek, P., Kozmik, Z. \& Busslinger, M. Chromosomal localization of seven PAX genes and cloning of a novel family member, PAX-9. Nat Genet 3, 292-298 (1993). 


\section{Acknowledgments}

This work was partly supported by grants from the Connecticut Stem Cell Research Program (10SCB12 and 12-SCB-UCON-02, to LL) and Fujian Innovative Medical Program (2012-cx-13).

\section{Author contributions}

M.S. designed and performed experiments, analyzed data, and wrote the manuscript. R.H., J.J., Y.Y. and Y.S. performed experiments and analyzed data, R.S. performed experiments. L. L. designed and performed experiments, analyzed data, supervised the study and wrote the manuscript.

\section{Additional information}

Competing financial interests: The authors declare no competing financial interests.

How to cite this article: $\mathrm{Su}, \mathrm{M}$. et al. Efficient in vitro generation of functional thymic epithelial progenitors from human embryonic stem cells. Sci. Rep. 5, 9882; DOI:10.1038/ srep09882 (2015).

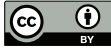

This work is licensed under a Creative Commons Attribution 4.0 International License. The images or other third party material in this article are included in the article's Creative Commons license, unless indicated otherwise in the credit line; if the material is not included under the Creative Commons license, users will need to obtain permission from the license holder in order to reproduce the material. To view a copy of this license, visit http://creativecommons.org/licenses/by/4.0/ 